\title{
Adjuvant Chemotherapy of Breast Cancer
}

\author{
Chair: Ulrike Nitz ${ }^{\mathrm{a}}$ \\ Participants: Fatima Cardoso ${ }^{b}$ Michael Gnant $^{c}$ Christoph Thomssen ${ }^{d}$ \\ a Brustzentrum Niederrhein, Evangelisches Krankenhaus Bethesda, Mönchengladbach, Germany \\ ${ }^{\mathrm{b}}$ Medical Oncology and Translational Research, Jules Bordet Institute, Brussels, Belgium \\ ${ }^{c}$ Abteilung für Allgemeinchirurgie, Universitätsklinik für Chirurgie MUW, Wien, Austria \\ ${ }^{d}$ Universitätsklink u. Poliklinik für Gynäkologie, Halle (Saale), Germany
}

\section{Question 1: Patients with NO Disease - Who Is Candidate for a Taxane and Which Regimen Should Be Given?}

Cardoso: Patients with high-risk node negative breast cancer have shorter survival than some patients with node positive disease. High risk may be defined by the presence of one or more of the following factors: hormonal receptors negative or low expression, grade 3 , HER2 positive, or high proliferation rate. Until we have a good predictive marker that allows us to choose between different cytotoxic agents we need to use both risk of recurrence and treatment safety (short- and longterm side effects) for our decisions. Therefore, and considering the higher cardiotoxicity risk of regiments with 6 cycles of anthracyclines, other regimens should be preferred, except maybe in some specific cases (see question number 4); these can be regimens with sequential administration of anthracyclines and taxanes (such as $3 \times \mathrm{FEC} \rightarrow 3 \times$ docetaxel or $4 \times \mathrm{E}(\mathrm{A}) \mathrm{C}$ dose-dense $\rightarrow 4 \times$ paclitaxel dose-dense or $4 \times \mathrm{E}(\mathrm{A}) \mathrm{C}$ $\rightarrow 12$ weeks of paclitaxel) or regimens with sequential administration of anthracyclines and CMF (4xepirubicine $\rightarrow 4 \times$ oral $\mathrm{CMF}$, especially in pre-menopausal women).

Gnant: In all available trials and meta-analyses, the relative benefit of taxanes in terms of hazard reduction is similar in node negative and node positive patients. Differentiation of regimens therefore follows mainly side effect concerns. Outside clinical trials, we would usually use a taxane based regimen in high-risk and node positive patients were adjuvant chemotherapy is indicated, in these cases, sequential treatment (PACS-01). In node-negative patients we use FEC $\times 6$.

Thomssen: The AGO guidelines answer Solomon-like: 'taxanes may be beneficial in N0 patients at high risk of recur- rence.' The NCCN Practice Guidelines in Oncology Breast Cancer leave it open, intermediate and high-risk node negative patients are assigned to category 1: chemotherapy regimens comprising taxane and non-taxane containing regimens. Thus, guidelines currently do not provide real help. Although data from 3 clinical trials are promising, overall survival benefit by adding taxanes has not been proven yet. Thus, data do not support the general use of taxane-containing therapy in node negative breast cancer. Moreover, risk groups that might benefit from taxane use are not well defined. The node negative breast cancer trial NNBC-3 Europe aimed at this question has finished recruitment with 4,150 patients earlier this year, data are not yet available. In conclusion, extrapolating from existing data, node negative patients with high risk of recurrence (e.g. G3) and those with estrogen receptor (ER) negative HER2 positive disease might benefit from adding taxanes. Although DocAC is the best studied regimen (GEICAM\#9805), most studies of other regimens included highrisk node negative disease; thus, the same taxane regimens as in node positive disease can be used.

\section{Question 2: Should Every Patient Routinely Receive an Anthracycline-Based Chemotherapy or Can We Successfully Identify Subgroups of Patients Who Benefit Only Marginally?}

Cardoso: At present, unfortunately we do not have any validated predictive marker to identify patients who respond or do not respond to anthracycline based chemotherapy. Topoisomerase II- $\alpha$ is not validated as a predictive marker; data on this marker are not consistent, with some results going in one direction and others in the opposite one; additionally it is not yet clear if protein or gene levels should be used. On the other

\section{KARGER}

Fax +497614520714

Information@Karger.de

www.karger.com
(C) 2009 S. Karger GmbH, Freiburg 
hand, anthracycline-based chemotherapy has a proven value in the adjuvant treatment of breast cancer patients and hence patients should not be denied these agents unless a specific contra-indication exists for their use.

Gnant: There will be subgroups of patients who will not markedly benefit from anthracyclines (compared to e.g. CMF) but they are not clearly defined. We will avoid anthracyclines in case of pre-existing cardiopathy. There are obviously patients who will not markedly benefit from any chemotherapy (high endocrine responsiveness and limited risk), these patients are not given cytostatic treatment.

Thomssen: Retrospective analyses of some studies show a marginal benefit of anthracyclines compared to CMF in patients with ER positive HER2 negative tumors. However, none of the potential predictive factors for the anthracycline effect has been prospectively confirmed. The EBCTCG overview demonstrated benefit from anthracyclines compared to CMF in all subgroups (N0/N+, ER+/ER-). To date, established standard with prospectively proven benefit is the use of anthracycline containing combination regimens. In patients with comorbidity, particularly pre-existing cardiac disease, benefit of anthracyclines must be weighted against potential harm. In those patients anthracycline-free therapy with a taxane, e.g. docetaxel / cyclophosphamide, might be a reasonable alternative.

\section{Question 3: Is There a Role for Dose-Dense or Dose- Intense Chemotherapy in Adjuvant Therapy and If Yes, Who Should Receive It?}

Cardoso: Yes, there is a role, however, patients should be carefully selected since these regimens are also more toxic and harder to tolerate especially in older patients. In principle, patients with hormonal receptor negative or high grade/ high proliferating tumors are the ones who seem to benefit the most from these approaches.

Gnant: We do not support dose dense/dose intense chemotherapy outside clinical trials, but recruit the majority of highrisk patients to trials where these approaches are used.

Thomssen: Data of CALGB \# 9741 demonstrated a benefit of dose-dense chemotherapy especially in node positive ER negative disease. The German AGO trial studying the dose-escalating and dose-dense ddETC regimen demonstrated the benefit for all subgroups independent of ER status. Dose-dense chemotherapy (ddETC) is a reasonable alternative in patients with $>3$ tumor infiltrated axillary lymph nodes. Actually, AC followed by weekly paclitaxel (ECOG \# 1199) has also characteristics of a dose-dense regimen - at least in the paclitaxel phase compared to the standard three-weekly schedule. This regimen is interesting for patients with $<4$ tumor infiltrated axillary lymph nodes, however, it seems to deliver similar efficacy as EC/AC followed by three-weekly docetaxel.

\section{Question 4: What Is the Optimal Chemotherapy Regimen in Triple Negative Breast Cancer?}

Cardoso: So far, there are no solid data to choose a different regimen for triple negative breast cancer in the adjuvant setting, i.e. anthracycline and taxane based regimens. Based on knowledge only from pre-clinical and advanced disease data, it may be wise to use more alkylating agents approved for the adjuvant setting such as cyclophosphamide (i.e. regimens such as $4 \times$ epirubicine $\rightarrow 4 \times$ oral $\mathrm{CMF}, 6 \times \mathrm{CMF}$ oral or i.v. days 1 and 8 , or $\left.6 \times \mathrm{F}_{100} \mathrm{EC}\right)$.

Gnant: Triple negative patients should as a matter of principle enter clinical trials, because it appears particularly important to improve our standard in this subgroup. When a patient is not suitable for a clinical trial, we will use our usual standard.

Thomssen: Actually, there are no convincing data that support the use of a specific regimen in triple negative disease. Particularly, no prospective clinical data exist to support the use of platinum complexes in these patients, although preclinical data seem to show some efficacy. Retrospective analyses of the PACS-01 study suggest benefit from docetaxel use in triple negative patients. Thus, today anthracycline-taxane combinations or sequences are used also in triple negative breast cancer.

\section{Question 5: What Is the Optimal Chemotherapy of HER2 Overexpressing Breast Cancer? What Is the Role of Anthracyclines?}

Cardoso: Again, there are no strong predictive factors that allow us to optimally choose the best agents and regimens for this subtype of breast cancer. However, there are some data suggesting that HER2 positivity may be associated with a better response to both anthracyclines and taxanes. Additionally, safety issues linked to the use of chemotherapy with anti-HER2 therapies in these patients, such as cardiotoxicity, must be taken into account. Therefore, the best regimens are sequential regimens of anthracyclines and taxanes, such as $3 \times \mathrm{FEC} \rightarrow 3 \times$ docetaxel or $4 \times \mathrm{E}(\mathrm{A}) \mathrm{C} \rightarrow 12$ weeks of paclitaxel. Only in patients with clear contraindications to the use of anthracyclines alternative regimens should be used such as PTH (paclitaxel + carboplatin + trastuzumab) or TC (docetaxel + cyclophosphamide).

Gnant: We are - as proposed by the St. Gallen Consensus not differentiating chemotherapy regimens because of HER2 status. Again, most HER2 patients will be offered trial participation, and all patients will receive adjuvant trastuzumab. 
Thomssen: Optimal chemotherapy in patients with HER2 overexpressing tumors should enable the start of trastuzumab as early as possible during the course of chemotherapy instead of sequential administration of trastuzumab after the end of chemotherapy. Also anthracyclines should be administered for having an additional effect in HER2-positive disease. Currently, my preference in patients with HER2 overexpressing tumors is the use of anthracycline-taxane sequences like EC followed by weekly paclitaxel or 3-weekly docetaxel with trastuzumab starting concomitantly with the taxane administration. However, the anthracycline free combination DocCbH seems to provide similar benefit and may be an alternative particularly in patients with cardiac risk factors or other situations where anthracycline use should be avoided.

\section{Question 6: Patients with Hormone Sensitive Disease - How Can We Identify Patients Who Can Be Spared Chemotherapy?}

Cardoso: Several tools can be used to attempt to identify the patients who might be spared adjuvant chemotherapy. 'Traditional' pathological characteristics such as high levels of expression of hormone receptors, low grade, and low proliferation are important factors. UPA and PAI1 are validated biological factors that can be used to identify these patients. Novel tools such as Oncotype DX and MammaPrint still need to be fully validated, which is being done in the prospective trials TailorX and MINDACT, respectively. Very importantly the balance between expected benefits and expected side effects must be discussed thoroughly with each individual patient and her preferences should be the driving force of the decision-making process.

Gnant: The majority of patients with hormone sensitive disease can be spared chemotherapy. High endocrine responsiveness, low grade, lack of lymphatic or vascular invasion, and limited size as well as no or minimal lymph node involvement are triggering chemo-free adjuvant therapy.

Thomssen: Basically, relative reduction of the risk of recurrence by adjuvant chemotherapy is independent from ER status and similar in ER-positive and ER-negative disease. The effect of chemotherapy also does not depend on the use of endocrine therapy. However, the absolute benefit is dependent on the underlying risk of recurrence that can only be estimated by the use of additional prognostic factors. Thus, in node negative breast cancer patients with well differentiated tumors (G1), low uPA/PAI-1 expression, possibly also high expression levels of ER and progesterone receptor $(\mathrm{PgR})$ and higher age, chemotherapy can be omitted.

\section{Question 7: What Chemotherapy Regimen Would You Propose in the Elderly with a Good Performance Status?}

Cardoso: Optimal geriatric assessment is essential in these patients. If this assessment does not identify important frailties and there are no other contraindications (for example high cardiac risk or high thromboembolic risk), these patients should receive the approved regimens for the adjuvant setting. Age in itself should not be a reason to withhold appropriate treatment. There are data from one randomized phase 3 trial that indicate that the TC (docetaxel + cyclophosphamide) regimen is a valid option for these patients. G-CSF support as primary prophylaxis should also be considered in elderly patients receiving adjuvant chemotherapy with either anthracyclines or taxanes.

Gnant: If the performance status is good, treatment does not differ from that for younger patients. In case of co-morbidities or frailty, an individual interdisciplinary decision will be made based on expected efficacy and tolerability balances, as well as quality of life issues. For some, capecitabine or vinorelbine based regimens may be an option.

Thomssen: Considering their life expectancy, elderly patients with a good performance status would potentially experience a recurrence of the disease. Thus, they benefit from adjuvant therapies that reduce their risk of recurrence or death from the disease. In order not to compromise their cardiac function, it might be considered to deliver non-anthracycline containing regimens to these patients (e.g. docetaxel / cyclophosphamide).

\section{Participants}

Fatima Cardoso, MD

Medical Oncology and Translational Research Jules Bordet Institute

Boulevard de Waterloo, 125, 1000 Brussels, Belgium

fatima.cardoso@bordet.be

Prof. Dr. med. Michael Gnant

Universitätsklinik für Chirurgie MUW

Abteilung für Allgemeinchirurgie

Währinger Gürtel 18 - 20, 1090 Wien, Austria

michael.gnant@meduniwien.ac.at

Prof. Dr. med. Christoph Thomssen

Zentrum f. Frauenheilkunde u. Geburtshilfe

Universitätsklink u. Poliklinik f. Gynäkologie

Ernst-Grube-Str. 40, 06097 Halle/Saale, Germany

christoph.thomssen@medizin.uni-halle.de 